\title{
Corela
}

Cognition, représentation, langage

HS-15 | 2014

Complémentarité des approches qualitatives et quantitatives dans l'analyse des discours

\section{Les genres du discours dans l'articulation des approches qualitatives et quantitatives}

\section{Thierry Guilbert}

\section{(2) OpenEdition}

Journals

Édition électronique

URL : http://journals.openedition.org/corela/3577

DOI : $10.4000 /$ corela. 3577

ISSN : 1638-573X

Éditeur

Cercle linguistique du Centre et de l'Ouest - CerLICO

Référence électronique

Thierry Guilbert, « Les genres du discours dans l'articulation des approches qualitatives et

quantitatives », Corela [En ligne], HS-15 | 2014, mis en ligne le 15 octobre 2014, consulté le 19 avril 2019. URL : http://journals.openedition.org/corela/3577 ; DOI : 10.4000/corela.3577

Ce document a été généré automatiquement le 19 avril 2019

\section{cc) (†) (อ)}

Corela - cognition, représentation, langage est mis à disposition selon les termes de la licence Creative Commons Attribution - Pas d'Utilisation Commerciale - Partage dans les Mêmes Conditions 4.0 International. 


\title{
Les genres du discours dans l'articulation des approches qualitatives et quantitatives
}

\author{
Thierry Guilbert
}

« D’emblée certaines objections de sociologues m'ont [...] attribué une volonté de dévaloriser les

méthodes quantitatives [...], tout au contraire,

j'avais voulu montrer la possibilité d'enrichir l'interprétation des résultats issus d'un traitement de données statistiques par l'analyse du contexte historique, toujours singulier, de leur construction et des variations qu'elles mesurent. "

J.-C. Passeron (2006:14)

1 L'objet de cet article est de réfléchir à l'articulation des approches qualitatives et quantitatives dans les analyses des textes et des discours ${ }^{1}$ (ATD) par le biais de la notion de genres du discours, laquelle fait partie du bagage théorique des analyses du discours (AD) dites qualitatives ${ }^{2}$. Il s'agit de montrer le double intérêt méthodologique et épistémologique que les approches quantitatives peuvent trouver dans l'intégration de cette notion de genres du discours et les bénéfices que ces dernières peuvent apporter en retour aux approches comparatives.

2 Je conserve ici la distinction qualitatif/quantitatif habituelle pour la démonstration malgré son caractère insatisfaisant - s'il existe des approches purement qualitatives, il est difficile de concevoir des approches purement quantitatives (Duchastel \& Laberge 2011). Dans les usages communs, les approches qualitatives sont définies en négatif comme des analyses non quantitatives, c'est-à-dire n'utilisant ni le comptage, ni les outils statistiques, quand les approches quantitatives renvoient aux ATD assistées par ordinateur. Cet article vise à dépasser la réfutation symétrique stérile - opposant la généralisation du quantitatif au singulier du qualitatif ou encore «l'abstraction chiffrée » 
à «l'anecdotique du vécu »-, il existe bien sûr des positions intermédiaires ou " mixtes » (Passeron 2006; Duchastel \& Laberge op. cit.). Ainsi, je partage le propos de Damon Mayaffre $(2002 ; 2004)$ selon lequel l'ordinateur est non seulement un outil probatoire, mais surtout un outil heuristique : s'il permet parfois de fournir des preuves objectives et quantifiées, il propose avant tout des pistes de réflexion au chercheur en lui faisant découvrir ce qu'il ne cherchait pas forcément.

Cela étant posé, on peut remarquer, empiriquement, que l'attrait pour l'outil informatique est souvent renforcé par le gage de scientificité que produit un résultat chiffré et par l'accessibilité des logiciels actuels à un public plus nombreux. Cet attrait dû peut-être à un sentiment d'infériorité des sciences humaines vis-à-vis des sciences dites objectives ${ }^{3}$ - est compréhensible mais occulte souvent un certain nombre de questions d'ordre général sur l'utilisation des chiffres dans les ATD dont celles-ci : la fonction probatoire est-elle l'apanage du quantitatif ? L'accès des sciences humaines à la scientificité passe-t-il par la seule production de données chiffrées? Quelle capacité le quantitatif a-t-il à produire du savoir hors études de cas ou encore à monter en généralité?

4 La question traitée ici s'inscrit dans ces questionnements épistémologiques en ce qu'il s'agit également d'une question souvent occultée : peut-on étudier un discours d'un point de vue quantitatif sans prendre en compte le genre discursif auquel ce discours appartient ? La réponse à cette question semble si évidente pour le linguiste que François Rastier peut affirmer : « Comme les traitements automatiques du langage ont affaire à des textes, non à des phrases, leur typologie est une condition de leur analyse » (2011: 71). Cette réponse peut sembler suffisante mais il semble que le sujet mérite d'être explicité plus amplement et je reformule donc la question ainsi : pourquoi l'analyse quantitative doit-elle prendre en compte le genre discursif, quelles en sont les raisons épistémologiques et méthodologiques?

5 Afin d'étayer ce questionnement, je m'appuierai sur une note de travail de Mikhaïl Bakhtine (1984a) figurant dans ses «Remarques sur l'épistémologie des sciences humaines $»^{4}$ et sur Le Raisonnement sociologique de Jean-Claude Passeron (2006) qui traite également de la question de l'épistémologie des sciences humaines en relation avec l'épistémologie des sciences expérimentales; j'opérerai ensuite un déplacement en rapportant ces éléments de réflexion à la notion de genres du discours afin de montrer combien certaines ATD quantitatives courent un «risque épistémologique» (von Münchow 2004) quand le genre y est un mal-pensé5, voire un impensé.

6 Cet article a donc une forme argumentative dans le but d'apporter des éléments de réflexion aux étudiants et aux chercheurs qui choisissent d'utiliser le nombre pour faire sens, selon la formule heureuse de D. Mayaffre (2011). La première partie présente succinctement la notion de genres du discours dans les sciences du langage et les relations qu'elle entretient avec l'analyse du discours, puis souligne les effets qu'implique l'usage de cette notion sur la définition du sujet et la place laissée au style individuel.

7 La seconde partie soulève un certain nombre de problèmes méthodologiques posés par l'intégration de cette notion, aux approches quantitatives. L'analyse du discours n'étant pas uniforme, je propose ensuite trois pistes permettant d'utiliser le genre dans les études articulant approches quantitatives et qualitatives dans le but soit de dresser des typologies, soit de décrire les discours, soit encore de les interpréter: la piste "générique » visant à définir les genres eux-mêmes, la piste «contrastive » faisant du 
genre l'invariant de la comparaison et la piste « interprétative » visant l'étude d'un genre donné.

\section{La notion de "genre du discours » dans les sciences du langage}

8 La notion de genre du discours, à ne confondre ni avec la notion des genres (morphologie linguistique ou flexion grammaticale), ni avec ce qu'on appelle genders studies dans les pays anglo-saxons, est présente depuis l'Antiquité, notamment grâce aux genres de la rhétorique (épidictique, délibératif et judiciaire). Dans la tradition classique, elle permettait de classer les productions écrites selon certains «textes-modèles " (BrancaRosoff, $2007: 115 \mathrm{sq}$.). Dans son usage courant actuel, elle est « un moyen pour l'individu de se repérer dans l'ensemble des productions textuelles » (Charaudeau, 2002: 277). En analyse du discours et en analyse textuelle, cette notion de genre discursif est appliquée à des textes non littéraires et plus largement à tous types de discours qu'ils soient oraux ou écrits ${ }^{6}$.

\subsection{Genre et historicité du discours}

Le terme " genre $d u$ discours » vient de Bakhtine ${ }^{7}$ (1984b) lequel, selon Charaudeau, a un « point de vue communicationnel» dans le sens où « les genres dépendent de la "nature communicationnelle" de l'échange verbal» (2002 : 279). En effet, Bakhtine précise que "l'utilisation de la langue s'effectue sous forme d'énoncés concrets uniques (oraux ou écrits) qui émanent des représentants de tel ou tel domaine de l'activité humaine " (1984b : 265) et ajoute une restriction d'importance quant à la liberté individuelle et à l'unicité des énoncés :

"Tout énoncé pris isolément est, bien entendu, individuel, mais chaque sphère d'utilisation de la langue élabore ses types relativement stables d'énoncés, et c'est ce que nous appelons les genres du discours. » (ibid. : 265, ital. de l'auteur)

10 La citation ci-dessus, définition bien connue des " genres du discours ", rappelle la nature sociale et prescriptive des genres du discours et peut être rapportée, du point de vue du dialogisme du discours, à une remarque de Bakhtine (1984a) à propos de l'épistémologie des sciences humaines. Selon l'auteur, les sciences exactes produisent une forme de savoir monologique parce qu'elles ne comportent qu'un seul sujet - le chercheur qui fait acte de cognition et qui parle ${ }^{8}$-, lequel étudie une "chose muette » qui n'est pas en interaction avec le chercheur. Plus : l'objet des sciences exactes reste une chose muette même quand il s'agit d'étudier l'homme car celui-ci n'est alors traité que comme une matière vivante, un objet. A l'inverse, dans les sciences humaines et sociales, la connaissance est dialogique puisque le sujet étudié, en sa qualité de sujet, n'est pas muet mais "répondant » (ibid.: 383) vis-à-vis du chercheur, des autres hommes et de son environnement - dont les institutions humaines. Les sciences humaines ont donc une forme d'historicité que l'auteur note ainsi: «Historicité. Immanence. Problème des frontières du texte et du contexte » (ibid. 384). Si le sujet est "répondant " dans les sciences humaines et sociales, c'est que le sens est à la fois dans le texte et le contexte, c'est-à-dire dans leur relation dialogique.

On retrouve une conception assez proche de celle du "sujet répondant » chez Passeron lorsqu'il précise ce qu'est l'historicité d'une discipline : « une discipline est historique dès 
que ses énoncés ne peuvent, lorsqu'il s'agit de les dire vrais ou faux, être désindexés des contextes dans lesquels sont prélevées les données ayant un sens pour ses assertions.» (2006: 153)

12 Ainsi, parce que son objet est d'étudier l'homme à travers les discours tenus dans des institutions, parce que son objet est un sujet, l'AD est une science historique, est indissolublement «associée à des répondants empiriques» (Maingueneau 2011:97) et prend acte d'un certain dialogisme du discours - même si, comme le remarque très justement Marie-Anne Paveau (2010: 147 sq.), tout discours n'est pas nécessairement dialogique.

13 Appliquée aux genres discursifs, cette conception du sujet répondant montre que ceux-ci possèdent des qualités " humaines », des qualités dynamiques de "sujet » : leurs racines sont historiques, géographiques et socioculturelles (Adam 2000), leur nombre n'est jamais fini (de nouveaux genres apparaissent constamment) et les genres existants sont soumis aux variations sociohistoriques. On voit alors combien cette notion tourne le dos à la tradition littéraire: les genres ne sont ni des modèles figés, ni des formes purement individuelles. Bakhtine note d'ailleurs, à propos du locuteur, que les genres «lui sont donnés, ce n'est pas lui qui les crée » (1984b : 287). Les genres sont des constructions temporelles, issues des interactions humaines et non des « choses muettes ». Plus : établir et percevoir un genre, c'est mettre des textes en rapport et les relier à leur contexte d'apparition, c'est la condition même de la compréhension effective - et de la production - d'un texte'.

14 Afin de spécifier les interrelations des genres avec le monde social, l'auteur distingue deux grands types de plans discursifs : les discours simples (ou discours premiers) et les discours complexes (ou discours seconds). Les productions «naturelles» (ou discours simples) appartiennent aux genres premiers qui ont un « rapport immédiat au réel existant et au réel des énoncés d'autrui » (1984b : 268). Ce sont les genres de la vie quotidienne, leur apprentissage inconscient se fait en même temps que l'apprentissage de la langue maternelle ${ }^{10}$ : «nous apprenons à mouler nos paroles dans les formes du genre » (ibid. 285). Les genres premiers sont de type « discours oral » - la langue des salons, des cercles, le langage familier - ce qui ne signifie nullement qu'il n'y ait de genres premiers écrits. Les productions « construites» (ou discours seconds) qualifiées de genres seconds (ibid.: 267) concernent plutôt les discours écrits, mais non exclusivement : ce sont les genres institutionnalisés comme les discours idéologiques, scientifiques, artistiques, littéraires, médiatiques...

Pour Bakhtine, cette distinction épistémologique est primordiale ${ }^{11}$ car tout chercheur qui ne tiendrait pas compte du genre et, plus précisément, du type de genre (premier ou second) courrait le risque d'un formalisme ou d'une abstraction excessive. On retrouve ici la conception du «sujet répondant»: en ne tenant pas compte de "la nature de l'énoncé ", on couperait en quelque sorte l'énoncé des conditions dans lesquelles celui-ci a été proféré et on dénaturerait l'historicité de l'étude, celle-ci perdrait alors tout contact avec le réel en affaiblissant le «lien entre la langue et la vie » (ibid.: 268). De la même façon, Passeron précise, dans sa réflexion sur l'épistémologie des sciences historiques, qu'" à mesure [...] que l'on avance dans [l']épuration statistique, le raisonnement expérimental s'améliore logiquement, mais devient, en même temps, de plus en plus absurde historiquement et, du même coup, sociologiquement. » (2006: 165)

L'un des moyens d'éviter ce risque lorsqu'on étudie des discours est alors de considérer la notion de genres du discours, institution humaine saturée de dialogisme, et de prendre en 


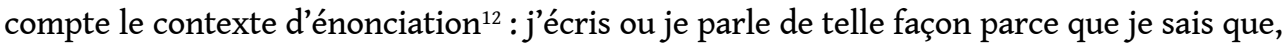
dans des circonstances analogues, on a parlé ou écrit comme cela avant moi et qu'on attend cela de moi. Bakhtine soutient ainsi, avec force et justesse, que ne pas tenir compte du genre c'est réifier le discours, donc réifier l'homme.

Cependant, les points de vue de Bakhtine, comme de Passeron, ne sont pas dogmatiques, il existe des « degrés de réification et de personnalisation » (1984a : 383-384), et il faut se garder des deux tendances extrêmes : la stricte empathie subjective de la « comparaison historique " et l'abstraction opaque de la "corrélation statistique " (2006: 160sq.). Passeron propose que les modalités de la preuve mises en œuvre dans les sciences humaines et sociales passent par:

\begin{abstract}
«la notion de "mixité" pour caractériser le raisonnement sociologique - va-et-vient argumentatif entre le raisonnement statistique et contextualisation historique - il est peut-être bon de préciser qu'une science située à mi-chemin entre deux démarches scientifiques n'est pas une science située à mi-chemin de la science. [... ] Le lieu [le locus épistémologique] où le raisonnement sociologique construit ses preuves et établit ses présomptions se situe ailleurs que dans l'espace argumentatif du raisonnement expérimental. » (2006:57 et 58)
\end{abstract}

La position essentielle de Passeron est que le « raisonnement sociologique » des sciences historiques, raisonnement scientifique différent du « raisonnement expérimental » est tout à fait admissible épistémologiquement dès lors que le chercheur exerce un certain contrôle réflexif sur son propre travail et une objectivation des représentations :

«Avec, pour seules armes de la "vigilance épistémologique" la critique de l'illusion de transparence, les techniques de rupture avec les pré-notions et l'exigence théorique de reconstruire en systèmes de relations les objets pré-construits par le sens commun » (ibid.: 66)

Il me semble que la théorie du discours de Michel Pêcheux rencontre les conceptions épistémologiques sur l'historicité de Bakhtine et de Passeron, et l'articulation des approches quantitatives et qualitatives avec Passeron ${ }^{13}$. La recherche par Pêcheux et Henry d'un modèle quasi mathématique (AAD) préfigurant les recherches quantitatives actuelles s'appuie avant tout sur la prise en compte de la notion de "conditions de production » : on peut y voir un effort d'objectivation et de formalisation des faits sociaux garanti toutefois des excès $\mathrm{du}$ formalisme par le «rappel au contexte historique » (Passeron, ibid.: 164-165) et le retour réflexif du chercheur sur son propre travail.

\title{
1.2 Style, genre et analyse du discours
}

Un certain nombre de points de convergence entre Bakhtine et Pêcheux (Guilbert 2010) justifient l'intégration déjà ancienne des genres du discours dans la « boîte à outils » de l'analyste du discours. Selon Dominique Maingueneau, l'analyste du discours « ne part d'un genre que pour l'inscrire dans ses lieux et ne délimite un lieu que pour considérer quel(s) genre(s) de discours lui sont associés » (1991: 13, ital. de l'auteur). Cette question des points de rencontre entre la notion de genres et l'analyse du discours est d'autant plus essentielle qu'elle est sous-tendue par une conception du sujet - j’y reviens (\$1.3).

21 Je voudrais auparavant éclaircir la nature de l'énoncé : si Bakhtine ne nie pas la notion de style individuel, il précise que le style est fonction du genre du discours dans lequel il est tenu. Le genre a une "valeur normative ${ }^{14}$ et impose les «formes prescriptives de l'énoncé » (1984b : 287). Par ailleurs, le style est plus ou moins individuel et plus ou moins 
effectif selon les genres; on admettra sans peine que tel genre littéraire est plus propice au style individuel que le genre « lettre officielle».

En AD, dès 1971, Haroche, Henri et Pêcheux ont relié directement la notion de «formations discursives » à ce que Bakhtine nomme genres du discours, même si ce lien n'est pas pensé et si cette catégorie ne semble pas essentielle pour les auteurs (Maingueneau 2011 : 89) :

Les « formations idéologiques [...] comportent nécessairement, comme une de leurs composantes, une ou plusieurs formations discursives interreliées, qui déterminent $c e$ qui peut et doit être dit (articulé sous la forme d'une harangue, d'un sermon, d'un exposé, d'un programme, etc.) à partir d'une position donnée dans une conjoncture donnée : le point essentiel ici est qu'il ne s'agit pas seulement de la nature des mots employés, mais aussi (et surtout) des constructions dans lesquelles ces mots se combinent, dans la mesure où elles déterminent la signification que prennent ces mots » (Haroche et al. 1971 : 102, ital. des auteurs)

«Ce qui peut et doit être dit» est donc déterminé par les formations discursives et est actualisé ("articulé ») sous la forme d'un genre du discours qui n'agit pas seulement sur le « contenu » du discours « mais aussi (et surtout) » sur la façon dont le propos est agencé et formulé. Chez Bakhtine également, la liberté d'action du locuteur est restreinte, le choix d'un genre est :

«fonction de la spécificité d'une sphère donnée de l'échange verbal, des besoins d'une thématique (de l'objet du sens), de l'ensemble constitué des partenaires, etc. Après quoi, le dessein discursif du locuteur, sans que celui-ci se départisse de son individualité et de sa subjectivité, s'adapte et s'ajuste au genre choisi, se compose et se développe dans la forme du genre donné. » (1984b : 284)

S'il ne s'agit pas d'assimiler ici les deux pensées - il y a, chez Pêcheux, la volonté, d'une part, de relier «le matérialisme historique et l'inconscient» (Maldidier et al. 1972:133) et, d'autre part, de pousser plus loin la pensée d'Althusser (1970) -, néanmoins le discours a, chez les deux auteurs, un rôle essentiel dans la diffusion des idéologies ${ }^{15}$, rôle que ne dément pas Volochinov : « la créativité idéologique, fait matériel et social », est le langage (1977 : 29). En termes bakhtiniens, il faut délaisser, dans l'étude des énoncés, le «sens monologique » pour le « sens dialogique » :

«Un énoncé doit être considéré, avant tout, comme une réponse à des énoncés antérieurs à l'intérieur d'une sphère donnée (le mot "réponse », nous l'entendons ici au sens large) : il les réfute, les confirme, les complète, prend appui sur eux, les suppose connus et, d'une façon ou d'une autre, il compte sur eux.» (Bakhtine $1984 \mathrm{~b}: 298)$

Si, pour Bakhtine, le style est un "épiphénomène» (1984b: 269) inscrit dans le dialogisme, parallèlement, chez Haroche et al., les discours sont contraints ou déterminés par les formations discursives, l'interdiscours et le « déjà-dit », lesquels laissent des traces dans les énoncés. Ces marques discursives montrent :

« la relation énoncé/énonciation, par laquelle le "sujet parlant" prend position par rapport aux représentations dont il est le support, ces représentations se trouvant réalisées par du "pré-construit" linguistiquement analysable » (1971: 106, ital. des auteurs).

Ainsi, quand l'AD restreint la part du style individuel ou de l'expression personnelle, elle pose la question de la «liberté du sujet parlant», autrement dit sa capacité d'agir librement dans les institutions (dont les genres discursifs) : la question du sujet. 


\subsection{Le genre et la question du sujet}

27 Cette question centrale en $\mathrm{AD}$ dans les années 70 est traitée ici à nouveaux frais en adoptant l'optique actuelle de Michel Wiewiorka qui, projetant de forger « un concept sociologique de la mémoire " lié à la constitution du sujet, cherche à dépasser les querelles passées ${ }^{16}$ :

«En fait, le problème pour être résolu doit être déplacé. Au lieu de partir d'un concept finalement confus de sujet pour rendre compte de l'action, ce qu'il importe de comprendre est l'aboutissement de processus au fil desquels se façonne le sujet, qui de là, à un instant donné, passera, ou non à l'action. Le sujet est alors, simultanément, ce que l'expérience ou l'action a fait, mais aussi d'ailleurs l'éducation et autres facteurs, et ce qu'il pourra faire, il est à la fois déterminé et déterminant. Le plus important n'est pas alors dans un concept nécessairement mouvant de sujet, il est dans la compréhension des processus qui conduisent à des états de sujet ${ }^{17}$ » $(2012: 6)$.

La position de l'AD est que le sujet n'est pas totalement libre quand il parle: les formations discursives et les genres du discours agissent sur, ou déterminent en partie, ses propos et sa façon de s'exprimer. Dans l'exercice quotidien du discours (genres premiers) comme dans l'usage des genres seconds ou institutionnalisés, le «sujet parlant » se trouve donc plongé dans des pratiques discursives socialement situées qui agissent comme des normes sociales (présentation de soi, prononciation ${ }^{18}$, registre de langue, arguments acceptables, etc.) : « ce qui peut et doit être dit ». Ces normes forment un "préconstruit linguistique », un déjà-dit, socialement intégré. Certains analystes du discours limitent toutefois la contrainte du genre au « comportement verbal » :

« [la] constatation d'instabilité, potentielle et relative des textes, considérés comme entrant dans un même genre discursif, par rapport au modèle dont ils procèdent, confirme le statut de présentation sociale partagée de la notion de genre : celle-ci agit comme une norme de comportement verbal et non comme une norme linguistique. » (Beacco 2004 : 114)

Il me semble au contraire que la contrainte du genre impose une norme discursive et une «norme linguistique »: ainsi les genres médiatiques comportent des traits linguistiques et énonciatifs communs indéniables ${ }^{19}$ dont on peut raisonnablement penser qu'ils constituent, au moins en partie, les traits d'un genre donné. Par exemple, les articles de commentaire (éditorial et chronique) de la presse écrite sont caractérisés en partie par le fait que les pronoms je et vous y sont quasiment absents alors que les pronoms nous et on y sont omniprésents (Guilbert 2007). Cependant, pour revenir à la question du sujet, il arrive que certains sujets s'affranchissent des normes discursives : ainsi Jean Daniel du Nouvel Observateur utilise fréquemment le je (Guilbert 2013b).

J'intègre donc le processus dialectique de Wieviorka en avançant que si le genre détermine en partie le sujet dans son dire, en retour, le sujet est déterminant pour le genre. Le sujet agit et, en agissant, il modifie et fait évoluer les genres du discours. C'est tout le propos de Branca-Rosoff $(2007: 112)$ qui s'intéresse au « problème de la frontière entre violation des normes et changement des normes", l'auteure rappelle ainsi que dans la tradition littéraire :

«Les normes du genre sont plurielles et les injonctions ou les interdits sont remplacés par l'adéquation à la situation moins contraignante [que les normes orthographiques]. La preuve en est que de nouvelles transgressions sont à la source de nouveaux genres [...]. En dernier lieu, c'est l'appréciation des récepteurs qui aura le dernier mot. » (ibid., $2007:$ :116) 
31 En résumé, les formations discursives - les médias, l'institution judiciaire, l'école, etc. configurent, par l'intermédiaire des sujets-acteurs que sont les locuteurs et dans des conditions sociohistoriques particulières, des matrices discursives qu'on appelle genres $\mathrm{du}$ discours: des types d'écrits (ou d'oraux) aux formes socio-discursives conventionnelles, c'est-à-dire fixées par la tradition, plus ou moins codifiées et dotées de caractéristiques distinctes et identifiables. En retour, les sujets-acteurs, inscrits dans des conditions sociohistoriques constamment mouvantes, confortent, transgressent et font évoluer ces genres.

32 C'est à ce point précis où se pose la question des critères définitoires à attribuer aux genres que les approches qualitatives et quantitatives sont complémentaires. Les approches qualitatives ont certes déjà posé un certain nombre de variables : il en est ainsi par exemple des trois facteurs croisés proposés par Charaudeau (1997) ${ }^{20}$. On pourrait y ajouter les éléments plus énonciatifs évoqués ci-dessus, comme le type de sujet énonciatif ou le degré de prise en charge du discours. Mais, les possibilités qu'offrent les logiciels actuels donnent aux approches quantitatives un rôle important dans la définition des genres et la mise au jour heuristique de critères nouveaux - j'y reviens dans la partie 2 .

\subsection{Le genre, garant de l'autorité discursive}

Une autre caractéristique sociologique de la notion de genre du discours doit être signalée ici, même si elle n'a pas de conséquence directe d'un point de vue purement quantitatif, car elle montre l'importance que les genres ont pour les locuteurs et permet à elle seule de justifier la prise en compte de la notion de genre par les études quantitatives. Le genre, en tant que produit sociohistorique, comporte en lui-même sa propre "légitimité » (au sens de Bourdieu). Les matrices discursives, en tant que "communication ritualisée ", sont une " "convention d'écriture" liée à un genre et pertinente en tant qu'elle "légitime le texte" qui se donne comme approprié à une situation de communication réglée » (Beacco 1992 : 12). Respecter les contraintes sociodiscursives d'un genre donné ${ }^{21}$ confère en retour au locuteur une forme de légitimité, c'est-à-dire « l'état ou la qualité de qui est fondé à agir comme il agit » (Charaudeau 2009 : 19).

Le respect des contraintes du genre, notamment pour ce qui concerne les genres seconds, fait preuve des compétences discursives du locuteur, il attribue au sujet le statut de «locuteur autorisé » et confère à son discours distinction et reconnaissance sociales. L'utilisateur d'un genre second a donc tout intérêt à se conformer aux contraintes du genre $^{22}$. Ceci constitue, s'il le fallait, un nouvel indice de l'intrication des institutions dans le discours et du discours dans les institutions.

Dès lors, les études quantitatives (et qualitatives) ne peuvent plus ignorer cet élément d'appréciation fondamental qu'est la composante genre du discours dans leurs comptages linguistiques non seulement parce que les sujets-acteurs se soumettent aux normes génériques discursives et linguistiques et que celles-ci doivent être prises en compte dans les analyses, mais également parce que les genres du discours sont caractérisés par des régularités potentiellement quantifiables et que les approches quantitatives peuvent jouer un rôle dans leur mise au jour. 


\section{Approches qualitatives/quantitatives et genres du discours}

\subsection{Le genre dans les approches quantitatives, une nécessité}

Les approches quantitatives s'intéressent par définition au quantifiable. Leur principe épistémologique dans l'administration de la preuve est donc de renforcer le degré de probabilité d'un résultat et/ou d'éprouver, en la validant ou en l'invalidant par le nombre, une intuition. Pour reprendre les termes de Passeron et de Bakhtine, les approches quantitatives des discours se situent du côté de l'«abstraction", de la "modélisation» et du «formalisme " tirant de faits discursifs épars des éléments statistiques incontestables et décontextualisés. Il n'y a là rien à redire, c'est la fonctionmême de cette approche, mais on voit de suite qu'une étude qui ne se bornerait qu'à cela pourrait, à juste titre, être qualifiée de "non-historique » ou de «dés-historicisée »: "l'historicité de l'objet est le principe de réalité de la sociologie ", écrit très justement Passeron (2006: 166). Toutefois il existe différents «degrés de réification et de personnalisation » et les approches quantitatives sont très souvent, et très heureusement, accompagnées d'une approche qualitative qui interprète les résultats obtenus en revenant au texte ${ }^{23}$ et au contexte d'énonciation. Ce processus d'interprétation est décrit ainsi par Beacco :

«L'acte linguistique d'interprétation consiste à démontrer la schématisation, comme produit/processus, en la décrivant, et à la localiser dans des formations discursives, de nature idéologique et historiquement constituées. » (2004:116)

Le retour au texte - "principe de réalité » - consiste à rapporter, grâce aux résultats obtenus par l'analyse quantitative, l'objet (le discours) à sa formation discursive car «tout critère purement linguistique (c'est-à-dire de type morphosyntaxique) est strictement insuffisant pour caractériser les processus [discursifs] inhérents à une formation discursive » (Pêcheux 1975 : 226, note 2, ital. de l'auteur) : les mots ne reçoivent leur sens que dans une formation discursive donnée.

Il me semble que, même si la notion de genre est absente du processus d'interprétation de Beacco, le même principe doit être retenu dans le rapprochement des résultats quantitatifs au genre étudié car on ne peut ignorer les processus discursifs inhérents à un genre donné. En effet tel genre discursif est constitutif de telle formation discursive et constitue un point d'articulation ou un médium (au sens de Mc Luhan) entre formations discursives et discours: si les formations discursives s'actualisent dans des genres discursifs et agissent sur « ce qui peut et doit être dit» dans des conditions données, les genres discursifs définissent à leur tour un «type d'énoncé relativement stable du point de vue thématique, compositionnel et stylistique » (Bakhtine, 1984b : 269).

Du point de vue de la réception, « comprendre un texte » (Bakhtine 1984a), c'est certes le replacer à sa formation discursive mais aussi le rapporter à d'autres textes, et notamment aux textes d'un même genre discursif.

41 La question est donc la suivante: quel crédit accorder aux résultats d'une étude quantitative/qualitative qui, ignorant le genre, croirait analyser le style d'un locuteur en ne sondant que les effets du genre sur le style de l'orateur, ou encore à une étude qui, croyant comparer les styles de deux locuteurs dans deux genres différents mesurerait les contraintes de chaque genre ? ${ }^{24}$ 
42 La méthodologie statistique ne peut sous-estimer l'existence théorique de cette donnée essentielle qu'est le genre du discours. En effet, délimiter la "population » statistique « avec soin, voire avec une rigueur méticuleuse » (Muller, 1992:11) consiste à définir à quel ensemble commun appartiennent les «individus " statistiques, les textes étudiés. Identifier par exemple le type de locuteur, son groupe social, ses habitus, etc., est nécessaire mais insuffisant: on s'en tiendrait alors uniquement aux formes de langue en ignorant le genre et ses effets discursifs. Ce serait non seulement une double erreur théorique - conférer au locuteur à la fois une créativité et une liberté totales et concevoir le sens indépendamment de ses conditions de production -, mais aussi une erreur méthodologique puisqu'on comparerait des « individus » non comparables.

\subsection{Quelques difficultés à dépasser} individualité, le chercheur doit donc déterminer, d'une part, ce qui est d'usage courant dans tel ou tel genre et, d'autre part, ce qui renvoie au style de l'individu.

45 - Il n'est pas toujours simple de distinguer les genres, ni aisé de préciser les spécificités d'un genre, d'autant que ceux-ci sont mouvants et que «l'explicitation des règles qui interviennent dans un genre n'en permet jamais une modélisation totale » (Branca-Rosoff 2007 : 117).

46 - Un autre risque serait de multiplier à tel point genres et sous-genres à propos d'un corpus donné qu'à chaque locuteur correspondrait un genre propre.

47 - Les préconstruits sont très nombreux dans les logiciels, les dictionnaires et le langage courant: il en va ainsi des genres comme des «registres de langue ", basés sur des classifications disparates et fortuites ${ }^{25}$.

48 - De la même façon que le corpus n'est pas un donné, il faut se garder d'une conception « naturaliste » ou « essentialiste » des genres et « ne pas se contenter d'échantillonner un réel déjà là » (Maingueneau 2011 : 98). Les genres sont donc autant à reconstruire par l'analyste qu'à reconnaître.

49 - A l'inverse, les genres « indigènes $»^{26}$ (au sens sociologique) ou endogènes ne sont pas forcément à rejeter, ils peuvent être pris comme indices des conditions de production imposées au locuteur (par exemple, la mention du genre dans le média).

Le point de vue du chercheur n'est pas celui des locuteurs, en effet ceux-ci se font une " représentation du contexte $»^{27}$ (Guilbert 2011) dans lequel ils se trouvent : ils n'ont pas forcément conscience d'utiliser tel ou tel genre - surtout lorsqu'il s'agit de genres premiers - et peuvent également confondre les genres (ironie/humour). La maîtrise des genres, la « compétence sémiolinguistique » (Charaudeau, 2009:26), n'est pas forcément la mieux partagée et n'a pas nécessairement à voir avec la connaissance de la langue (Bakhtine, 1984b : 286-287) mais plutôt avec la diversité des expériences vécues. 


\subsection{Trois exemples d'approche qualitative/quantitative des genres}

\subsubsection{Pour une définition quantitative des genres du discours} personne permettant de comprendre comment se constitue le sujet énonciatif d'un genre scientifique (Poudat 2006) ou d'un genre médiatique ${ }^{28}$. Il est également possible de comptabiliser la ponctuation, les temps et modes verbaux, les catégories et modalités verbales $^{29}$, les classes morphosyntaxiques, les thèmes... et de spécifier ainsi les règles de prédilection d'un genre.

52 L'approche quantitative a donc un rôle précieux à jouer dans la définition linguistique précise et statistiquement argumentée des genres du discours, «indigènes » ou non. Rastier propose la méthodologie suivante pour dresser une typologie des genres :

«Nous chercherons [...] si une classification descendante est confirmée par une classification ascendante, de manière à parvenir à une classification objectivée. Si les classes formées par la classification automatique recoupent les genres prédéfinis, cela confirmera le caractère déterminant des genres sur les usages linguistiques. » $(2011: 77)$

S'appuyer sur l'approche quantitative permet au chercheur de réduire le risque de naturalisation les genres et de reconstruire les genres en comparant les spécificités observées dans le corpus aux préconstruits du chercheur et non l'inverse. On trouve la même approche chez Céline Poudat, « la caractérisation d'un genre ne peut se fonder que sur un corpus. La démarche est donc nécessairement empirique et ascendante » (2006:

37) car on ne peut prédire à l'avance ce que seront les caractéristiques d'un genre :

"L'étude des genres doit être ainsi objectivée, de telle sorte que le quantitatif puisse participer à la qualification des genres. [...] Etant donné que les méthodes quantitatives permettent de prendre en compte de nombreux critères de niveaux différents et d'éprouver leurs relations afin de faire émerger des phénomènes non, voire contre-intuitifs, il nous semble qu'elles doivent constituer un préalable à toute étude générique. » $(2006: 39)$

\subsubsection{La prise en compte du genre dans deux types d'approches contrastives}

L'analyse de textes assistée par ordinateur a une vertu heuristique, non seulement parce qu'elle permet de montrer au chercheur des éléments qu'il n'aurait pas perçu, mais aussi parce l'outil informatique et, plus précisément, statistique permet la comparaison des corpus tout en proposant des formes de présentation souvent très éclairantes (analyse arborée, analyses factorielles, graphiques, tableaux, hapax, spécificités, etc.). Cet autre secteur, dans lequel peut intervenir avec profit l'approche quantitative, est donc le 
champ des approches contrastives ou comparatives qui est autant qualitatif que quantitatif.

La notion de genre du discours semble ici incontournable pour écarter le risque d'artefact ${ }^{30}$. Ainsi, pour prendre un exemple quelque peu caricatural, comparer la constitution du sujet énonciatif de deux hommes politiques en comptabilisant les déictiques de la personne utilisés, sans tenir compte que l'on mélange interviews de l'un et allocutions officielles de l'autre, risque de montrer que le premier est plus autocentré que l'autre : ce résultat serait un artefact car on aurait mesuré non pas les spécificités du locuteur mais les récurrences génériques du genre interview qui induit une sur-utilisation du je (Denis 1989).

L'analyste doit prendre quelques précautions pour éviter cet écueil. Le principe est inverse au type d'étude proposé précédemment puisqu'il s'agit ici non pas de s'intéresser au genre pour lui-même mais de neutraliser les effets des contraintes du genre sur les résultats chiffrés. Pour ce faire, le genre discursif doit donc être déterminé en amont de l'étude, lors de la constitution du corpus, comme le tertium compartionis ou l'invariant de la comparaison (von Münchow 2004 ; Poudat 2006). Toutefois, on bute sur une des difficultés épistémologiques soulevées ci-dessus : l'« immanence » des genres. Constituer un corpus en se basant sur des genres institués a priori ou prédéfinis comporte effectivement le risque de naturaliser les représentations des acteurs. Deux attitudes, qui forment autant de pistes, semblent alors possibles pour prévenir ce risque.

La première piste consiste à refuser toute immanence des genres discursifs et donc à procéder en deux temps: tout d'abord redéfinir le genre en question en vérifiant quantitativement ses spécificités, puis procéder à l'étude contrastive afin de mesurer, par exemple, les différences de style de deux ou plusieurs locuteurs. Ce type d'approche s'intéresse à l'individualité des styles et des locuteurs dans une démarche plutôt philologique, voire littéraire (Labbé \& Labbé 2009). Dans le livret accompagnant Hyperbase , Etienne Brunet indiquait ainsi que « les informations nouvelles » que donnent les « bases transversales et synthétiques" sont " comparatives et quantitatives. Elles sont d'autant plus précieuses que le genre littéraire est un champ de recherche encore peu exploité où l'on peut s'attendre à des surprises et des découvertes" (2002: 102). Cette approche permet d'étudier également les variations de style d'un point de vue qualitatif dans le discours télévisuel (von Münchow 2004), comme d'un point de vue quantitatif/qualitatif dans le discours politique (Mayaffre 2004) ou dans les registres de condoléances: « Pour reprendre les catégories de Simmel, les messages de condoléances sont aujourd'hui une "forme" qui accepte un grand nombre de "contenus" différents "; même si le cadre théorique est différent, les préoccupations de Gérôme Truc (2011 : 30) semblent proches.

La seconde piste, adoptée notamment dans mes travaux, consiste à définir les genres d'un point de vue qualitatif et socio-discursif - statut de l'instance, mode discursif, degré d'engagement (Charaudeau 1997) -, en s'appuyant dans un premier temps sur le point de vue partagé des sujets et des institutions, dont les mentions dans les journaux. Ce n'est que dans un second temps que l'on mesure, de façon qualitative/quantitative et contrastive, les spécificités discursives d'une publication. L'objectif est alors très différent de l'attitude précédente puisqu'il s'agit d'étudier, par l'usage qu'ils en font, les représentations des sujets à propos du genre institutionnel en question et d'analyser comment fonctionne le discours diffusé au public à travers celui-ci. Lorsque les effets du genre sont neutralisés, on peut, comme l'écrit par exemple Wim Remysen, «analyser comment le discours normatif est construit dans les chroniques de langage » (2011:52) 
ou encore analyser comment fonctionnent les modalités persuasives et pragmatiques d'un genre médiatique. Cette méthode m'a permis de montrer (Guilbert 2007; 2013a ; 2013b) que l'éditorial et la chronique produisent un paradoxe pragmatique : malgré leur fort degré d'engagement et leur fonction sémiologique de commentaire, ils n'utilisent pas l'argumentation explicite mais l'effet d'évidence, notamment par la désubjectivisation du sujet et la co-responsabilisation du dire, pour persuader le lecteur.

\section{Conclusion} combinent harmonieusement dès lors que l'on tient compte d'un certain nombre de prérequis, en particulier la prise en compte de la notion de genres du discours par les analyses de textes assistées par ordinateur ${ }^{31}$. mélanger les populations statistiques, mais aussi admettre que les locuteurs contrôlent parfaitement leur dire, qu'ils ne sont en aucune façon déterminés par les structures dans lesquelles ils évoluent. L'étude perd alors contact avec le réel et court un double risque d'artefact et de réification de son objet.

On peut toutefois choisir de s'affranchir des genres ${ }^{32}$ en les neutralisant, mais cela ne peut se faire qu'en connaissance de cause, qu'en prenant en compte les spécificités et contraintes génériques qui jouent sur les pratiques discursives de leur utilisateurs.

Les pistes proposées nécessitent ainsi un retour constant au texte et à ses extérieurs constitutifs (contexte d'énonciation, formations discursives et textes d'un même genre), retours essentiels pour une interprétation et une compréhension effectives. Si les genres du discours «reflètent le moindre changement dans la vie sociale» (Bakhtine, 1984b: 271), la reconnaissance de la récurrence générique constitue alors le garant épistémologique des études contrastives et une avancée bénéfique pour les approches quantitatives comme pour les approches qualitatives.

\section{BIBLIOGRAPHIE}

Adam J.-M., 2000. Genres de la presse écrite et analyse de discours, Semen 13. 7-14.

Adam J.-M., 2001. Linguistique textuelle. Des genres de discours. Paris : Nathan.

Althusser L., 1976 [1970]. Idéologie et appareils idéologiques d'Etat. Notes pour une recherche. In Positions (paru dans La Pensée 151, 1970). Paris : Editions Sociales. 67-125.

Bakhtine M., 1984a [1974]. Remarques sur l'épistémologie des sciences humaines. In Esthétique de la création verbale. Paris : NRF, Gallimard. 379-393.

Bakhtine M., 1984b [1979]. Les genres du discours. In Esthétique de la création verbale. Paris : NRF, Gallimard. 263-308.

Corela, HS-15 | 2014 
Beacco J.-C., 1992. Les genres textuels dans l'analyse du discours : écriture légitime et communautés translangagières. Langages 105. 8-27.

Beacco J.-C., 2004. Trois perspectives linguistiques sur la notion de genre discursif. Langages 153. 108-119.

Berthoud-Papandropoulou I. \& Kilcher H., 2004. Acquisition du langage et genres énonciatifs. Langages 153. 52-61.

Biber D., 1988. Variation across speech and writing. Cambridge University Press.

Bourdieu P., 2001. Langage et pouvoir symbolique. Paris : Seuil.

Branca-Rosoff S., 2007. Normes et genre de discours, le cas des émissions de libre antenne sur les radios jeunes. Langage et société 119. 111-128.

Brunet E., 2002. Hyperbase. Manuel de référence. Institut de linguistique française/CNRS.

Charaudeau P., 1997. Le discours d'information médiatique. La construction du miroir social. Paris : Nathan-INA.

Charaudeau P., 2002. Entrée « genres ». In Charaudeau P. \& Maingueneau D., (éds), Dictionnaire d'analyse du discours. Paris : Seuil.

Charaudeau P., 2009. Identité sociale et identité discursive. Un jeu de miroir fondateur de l'identité langagière. In P. Charaudeau (éd.), Identités sociales et discursives du sujet parlant. Paris : L'Harmattan. 15-28.

Denis V., 1989. L'interview dans la presse écrite : une interaction représentée. Thèse de doctorat, Université de Rouen.

Duchastel J. \& Laberge D., 2011. La mesure comme représentation de l'objet. Analyse et interprétation, in SociologieS, avril, http://sociologies.revues.org.

Gadet F., 2010. Enjeux de langue dans l'analyse du discours. Semen 29, 111-123.

Guilbert T., 2007. Le discours idéologique ou la force de l'évidence. Paris : L'Harmattan.

Guilbert T., 2010. Pêcheux est-il réconciliable avec l'analyse du discours ? Une approche interdisciplinaire. Semen 29. 127-139.

Guilbert T., 2011. La représentation du contexte dans le discours. In T. Guilbert \& L. Baugnet (éds), Discours en contextes. Paris : PUF. 157-181.

Guilbert T., 2013a. La " réalité » de la presse écrite et l'éthique de l'analyse du discours. In C. Guérin, G. Siouffi et S. Sorlin (éds), Le rapport éthique au discours. Peter Lang. 298-302.

Guilbert T., 2013b. La « mise en évidence » du discours économique par la presse écrite. In M. Temmar, J. Angermüller \& F. Lebaron (éds), Les discours de l'économie. Paris : PUF. 139-158.

Guilbert T., 2013c. Evidence discursive et idéologie. Réflexion anthroposociale sur la constitution des représentations économiques partagées dans les discours de presse. Synthèse d'habilitation à diriger les recherches, Paris 13.

Haroche C., Henry P. \& Pêcheux M., 1971. La sémantique et la coupure saussurienne : langue, langage, discours. Langages 24. 93-106.

Labbé C. \& Labbé D., 2009. Existe-t-il un genre épistolaire? Hugo, Flaubert et Maupassant. Communication aux journées de l'ERLA. Brest : 20-21 novembre 2009. 
Labbé C. \& Labbé D., 2010. La modalité verbale en français contemporain. Les hommes politiques et les autres. Communication aux journées de l'ERLA. La modalité dans le texte de spécialité. Brest : 19 novembre 2010.

Maingueneau D. \& Cossutta F., 1995. L'analyse des discours constituants. Langages 117. 112-125.

Maingueneau D., 1991. L'analyse du discours. Paris : Hachette supérieur.

Maingueneau D., 2004. Typologie des genres institués. Version remaniée des pages 180-187 du Discours littéraire. Paris, A. Colin, 2004, disponible sur le site http://pagespersoorange.fr/ dominique.maingueneau/intro_topic.html

Maingueneau D., 2011. Pertinence de la notion de « formation discursive » en analyse de discours. Langages et société 135. 87-99.

Maldidier D., Normand, C. \& Robin, R., 1972. Discours et idéologie : quelques bases pour une recherche. Langue Française 15. 116-142.

Mayaffre D., 2002. L'Herméneutique numérique. L'Astrolabe (Ottawa), 14 p., www.uottawa.ca/ academic/arts/astrolabe/articles/art0031.htm, consulté en avril 2006

Mayaffre D., 2004. Paroles de président. Jacques Chirac (1995-2003) et le discours présidentiel sous la Vème République. Paris : Honoré Champion.

Mayaffre D., 2011. Quand le nombre fait sens. Revue Tranel 55. 95-109.

Mc Luhan M., 1968 [1964]. Pour comprendre les média. Paris : Seuil.

Moirand S., 2003. Quelles catégories linguistiques pour la mise au jour des genres de discours. Journée d'étude sur les genres de l'oral (UMR GRIC-Lyon 2), le 18 avril 2003, actes consultables : http://gric.univlyon2.fr/Equipe1/actes/journees_genres.htm

Muller C., 1992 [1973]. Initiation aux méthodes de la statistique linguistique. Paris : Champion.

Passeron J.-C., 2006. Le raisonnement sociologique - Un espace non poppérien de l'argumentation, 2 ème édition revue et augmentée par l'auteur. Paris : Albin Michel.

Paveau M.-A., 2010. La norme dialogique. Positions critiques en philosophie du discours. Semen 29. 141-159.

Pêcheux M., 1969. Analyse automatique du discours. Paris : Dunod.

Pêcheux M., 1975. Les vérités de La Palice. Linguistique, sémantique, philosophie. In Maldidier F., 1990. L'inquiétude du discours. Edition des cendres.

Poudat C., 2006. Etude contrastive de l'article scientifique de revue linguistique dans une perspective d'analyse des genres. Thèse de doctorat, Université d'Orléans, http://www.texto-revue.net

Ramysen W., 2011. L'application du modèle de l'Imaginaire linguistique à des corpus écrits : le cas des chroniques de langage dans la presse québécoise. Langage et société 135. 47-65.

Rastier F., 2011. La mesure et le grain. Sémantique de corpus. Paris : Champion.

Truc G., 2011. Analyser un corpus illisible ? Le logiciel Alceste confronté à des registres de condoléances. Langage et société 135. 29-45.

Van Dijk, T., 2006. Politique, Idéologie et Discours. Semen 21. 73-102.

Volochinov V. N., 1977 [1929]. Le Marxisme et la philosophie du langage. Paris : Minuit.

Von Münchow P., 2004. Les journaux télévisés en France et en Allemagne. Plaisir de voir ou devoir de s'informer. Paris : Presses de la Sorbonne nouvelle. 
Wieviorka M., 2012. Du concept de sujet à celui de subjectivation/dé-subjectivation. FMSHWP-2012-16, juillet, 15 p.

\section{NOTES}

1. Cette formule désigne les disciplines qui s'intéressent aux discours ; l'analyse du discours (AD) en est un sous-ensemble.

2. Par exemple la revue Semen $n^{\circ} 13$ (2000), Adam (2001), Moirand (2003), la revue Langages $n^{\circ} 153$ (2004) ou plus récemment la journée Conscila « Discours rapportés et genres de discours » (ENS, 2010) ou le colloque « Interpréter selon les genres » (Marrakech, 2013).

3. Sentiment résumé par deux questions qui ouvrent la préface de Passeron : «Science ou non? Si oui comme les autres ou pas?» ([1991] $2006: 27)$. L'auteur traite de la sociologie, mais j'élargis ces questions aux ATD.

4. Ses derniers écrits (1974), notes de travail reprises d'une étude de 1940, traitent des «fondements philosophiques des sciences humaines» sous forme de bribes, de paragraphes ou de pages entièrement rédigées.

5. Rastier (chap. 3, 2011) montre que certains logiciels utilisent, non sans problèmes, la notion. Il est intéressant de noter que la réflexion est menée par un « non-quantitativiste ».

6. Par exemple, Branca-Rosoff (2007) s'intéresse aux genres oraux dans les «émissions de libre antenne sur les radios jeunes ». Gadet (2010) revient sur la distinction oral/écrit en montrant que les genres dépassent largement celle-ci.

7. L'approche bakhtinienne est classique en $\mathrm{AD}$, même si d'autres approches existent comme celle de D. Biber (1988) qu'on peut retrouver in Poudat (2006), Gadet (2010) ou Rastier (2011).

8. Ce sujet n'est pas totalement seul puisque ses énoncés s'insèrent dans un discours scientifique. Cette remarque n'invalide en rien la démonstration de Bakhtine.

9. «La compréhension est une mise en rapport d'un texte aux autres textes. Le commentaire. Dialogicité de cette mise en rapport. [...] Comprendre c'est mettre en rapport aux autres textes et penser dans un contexte nouveau » (1984a : 384).

10. Berthoud-Papandropoulou et al. (2004) élargissent la notion de genre discursif aux «genres énonciatifs » qui s'acquièrent dès la petite enfance.

11. «Une conception claire de la nature de l'énoncé en général et des types variés d'énoncés en particulier (premiers et seconds), c'est-à-dire des divers genres du discours, est indispensable à toute étude quelle qu'en puisse être l'orientation spécifique. » (1984b : 268)

12. Contexte d'énonciation englobe ici la situation de communication et la situation d'énonciation, de même que conditions de production et de réception du discours (au sens de Pêcheux).

13. On aura reconnu dans la citation ci-dessus certains de ses termes de prédilection (« illusion de transparence ", " préconstruits », « sens commun »), même si l'ironie cible certainement plus Bourdieu que Pêcheux.

14. Mc Luhan (1968) a montré d'une autre façon cette détermination : «Le message, c'est le médium ».

15. Voir aussi Fairclough (1995) et Teun Van Dijk (2006: 83) qui citent d'ailleurs Pêcheux.

16. De façon rapide, l'optique marxiste (un sujet totalement déterminé par la structure de la société) s'opposait à la tradition libérale (un sujet entièrement libre et raisonnable). Je reviens plus longuement sur la question du sujet dans la synthèse de mon habilitation à diriger les recherches (2013c, 5.2 « Le sujet et l'événement »). Ces querelles sont encore très présentes dans les débats entre philosophes et analystes du discours aujourd'hui.

17. Wieviorka évoque les états de « sujet flottant », d' " anti-sujet » et de «non-sujet » (2012:5).

18. Voir les travaux du sociolinguiste W. Labov dans les années 60-70 au niveau phonologique notamment. 
19. Sauf à considérer que Beacco (2004) intègre les marques énonciatives dans le « comportement verbal ", il n'en reste pas moins qu'une étude quantitative pourra traiter ceux-ci comme des marques (et des normes) linguistiques du genre.

20. L'origine de l'instance énonciative, le degré d'engagement, le traitement de l'événement (commenté, provoqué, rapporté).

21. Bakhtine remarque qu'à l'oral, les «genres élevés» sont stables et "prescriptifs (normatifs) », le « vouloir dire » se limite uniquement aux nuances intonatoires (1984b : 286).

22. On pense ici à l'homme politique, à l'avocat ou au journaliste qui doit faire preuve des compétences discursives (et génériques) attendues. Même s'il viole ces règles, il doit montrer, afin de ne pas paraître illégitime, qu'il le fait en toute connaissance de cause.

23. Texte a une acception linguistique ici : production orale ou écrite non "préparée » pour l'ordinateur.

24. Adam remarque également «le caractère pragmatiquement nécessaire des genres », "la catégorisation générique même vague d'un objet discursif en permet la production autant qu'elle en guide la lecture » (Adam 2000, 8).

25. Bakhtine (1984b : 270). Il est à noter que l'auteur ne distingue pas précisément les genres du discours des registres, à la différence de Maingueneau (2011:91-92).

26. Maingueneau (2011 : 91-92) distingue « unités topiques » (que j'appelle indigènes) et «non topiques » (« construites par le chercheur »).

27. Celle-ci, basée sur les cadres de perception, peut être non consciente et elle est à prendre en compte par le chercheur.

28. Par exemple, «nous » et « on » représentent plus de $95 \%$ des occurrences du sujet énonciatif dans les éditoriaux et chroniques du Point lors du commentaire des manifestations contre la « réforme des régimes spéciaux des retraites » en 2010 alors que le « je » et le « tu » représentent respectivement $1,87 \%$ et $0 \%$ (Guilbert 2013a).

29. Par exemple, le travail de Labbé et Labbé (2010) ne porte pas sur un genre précis mais cherche à définir par le quantitatif l'utilisation de la modalité verbale (auxiliation de modalité) dans un type de discours donné.

30. La polémique Molière/Corneille est relancée en 2003 par le travail lexicométrique de Cyril et Dominique Labbé critiqué notamment pour sa prise en compte insuffisante du genre " comédie » et des traits propres à un autre genre, la Commedia dell'Arte (voir par exemple Wikipédia : Paternité des œuvres de Molière).

31. La remise en question (Maingueneau et al. 1995) de la notion de "support » en est un autre. Elle n'est pas sans rapport avec ce sujet mais la place manque ici pour l'aborder.

32. En travaillant sur «la circulation des signifiants» (Maingueneau 2011: 93) et, j'ajouterais, l'intertextualité.

\section{RÉSUMÉS}

Cet article vise à montrer comment la notion de genres du discours permet d'articuler les approches qualitatives et quantitatives. Il en expose les raisons épistémologiques et les implications méthodologiques, notamment la conception du sujet qu'elle suppose et les risques d'artefact qu'il y a à ne pas penser la notion de genres du discours. Il montre enfin combien les 
approches quantitatives sont utiles, en retour, pour définir et neutraliser les genres dans les études comparatives.

This article aims to explain how the notion of speech genres can articulate qualitative and quantitative approaches. It outlines epistemological reasons and methodological implications, notably the definition of the subject that it assumes and the risks of artefact to not think the notion of speech genres. It shows finally how quantitative approaches are useful, in return, for the definition and the neutralization of genres in comparative studies.

INDEX

Mots-clés : analyse du discours, genres du discours, sujet répondant, épistémologie, études comparatives, approches qualitatives et quantitatives

Keywords : discourse analysis, speech genres, responding subject, epistemology, comparative studies, qualitative and quantitative approaches

\section{AUTEUR}

\section{THIERRY GUILBERT}

Université de Picardie Jules Verne CURAPP-ESS (UMR 7319) 